\title{
Non Orthogonal Multiple Access Technique in 5G
}

\author{
Mayank Kothari \\ Assistant Professor (M.Tech) \\ Department of Electronics \& Telecommunication \\ Engineering \\ SVKMs Narsee Monjee Institute of Management \\ Studies (Deemed-to-be UNIVERSITY), Mukesh Patel \\ School of Technology Management \& Engineering, \\ Shirpur, India
}

\author{
Pravin Wararkar, PhD \\ Assistant Professor \\ Department of Electronics \& Telecommunication \\ Engineering \\ SVKMs Narsee Monjee Institute of Management \\ Studies (Deemed-to-be UNIVERSITY), Mukesh Patel \\ School of Technology Management \& Engineering, \\ Shirpur, India
}

\begin{abstract}
Non-Orthogonal Multiple Access is a promising radio access system for execution redesign in bleeding edge cell correspondences. Still out from Symmetrical repeat division diverse access, which is an eminent high-limit even unique access methodology, it offers a great deal of appealing focal points, including increasingly noticeable range viability. Is different sorts of this techniques, including power-region and code-space. This paper focuses on a very basic level revolves around power-space NOMA that utilizes superposition coding at a transmitter and dynamic impedance crossing out at a authority. Various experts have displayed that NOMA is used effectively till meet both framework level and experienced data rate essentials of fifth-generation headways. Starting is of view, this paper in audits a continuous headway of NOMA in 5 Generation structures, looking over a tier limit examination, power task frameworks, goodness, and coordinating plans in NOMA. In addition, this paper discusses how NOMA performs when it is composed with various showed remote exchanges methodologies, for instance, pleasing trades, various data different yield (MIMO), pillar framing, space-time coding, and framework coding, among oars. In addition, this paper discusses a couple of critical issues on NOMA utilization and gives a couple of streets till future research.
\end{abstract}

\section{Keywords}

NOMA, MIMO

\section{INTRODUCTION}

From simple calls through till all IP management, including voice and informing, each progress has been urged by a need till meet prerequisites of a new age of portable innovation. Along as lines, portable correspondences innovation is by and by confronting an oar test, bringing forth a hyper-associated society through a rise of fifth-age management. With tremendous potential for a two shoppers and industry, 5 Generation is relied upon till turn out by 2021. From a cutting edge radio access innovation perspective, a stage change in details speed and a critical decrease in start till finish inertness is a significant worry for $5 \mathrm{G}$, since a fast advancement of a versatile Internet and an Internet of Things exponentially quickens an interest for high details rate applications. Specifically, a large number of a business activities that have advanced with take a shot at 5 generation pronounces that is system level details rate in $5 \mathrm{G}$ ought till be 10-20 Gaps (that is, 10-20 times a pinnacle information rate in $4 \mathrm{G}$ ), and a client experienced details rate ought till be 1 Gaps (multiple times a client experienced details rate in $4 \mathrm{G}$ ). An additionally set inertness (start till finish full circle delay) at 1 millisecond (one-fifth of a dormancy in 4G). A hidden physical association in a phone arrange is called radio access innovation, which is executed by a radio access organize.

A RAN essentially uses a channel get till method till give versatile terminals an association with a center system. A structure of an appropriate numerous entrance methods is one of most significant viewpoints in improving a framework limit. Numerous entrance methods can extensively be ordered two unique approaches, in particular, symmetrical different access and non-symmetrical numerous entrance. A symmetrical plan permits an ideal beneficiary till isolate undesirable signs from an ideal sign utilizing diverse premise capacities. At an end of a day, signals from various clients are symmetrical till one an oar in symmetrical

plans. Time division numerous entrance, and symmetrical recurrence division numerous entrance is two or three instances of OMA plans. In TDMA, a few clients she's a similar recurrence channel on a period sharing premise. Clients convey in quick progression, in a steady progression, each utilizing air allocated availabilities. OFDMA permits multi-client correspondences through a symmetrical recurrence division multiplexing method in which subcarrier frequencies is picked with a goal that subcarriers is symmetrical till one another. Rather than OMA, NOMA permits allotting one recurrence channel till different clients simultaneously inside a similar cell and offers various focal points, counting improved unearthly productivity, higher cell-edge throughput, loosened up channel criticism (just a got signal quality, not precise channel state data, is required), furthermore, low transmission inactivity (no booking demand from clients till base station is required). Accessible NOMA systems can extensively be isolated into two classifications, till be specific, power what's more, code NOMA. This paper centers around a force space NOMA that superposes various clients in power space and adventures a channel gain distinction between multiplexed clients. At a transmitter side, signals from different clients are superposed and a subsequent sign is at that point transmitted over similar channels (i.e., a similar time-recurrence assets). At sides, multiuser identification calculations, for example, progressive impedance abrogation is used till recognize an ideal signs. In spite of a fact that this paper essentially overviews a force space superposition coding based NOMA, a short conversation of different classes of NOMA is surrendered in a following segments.

\section{NOMA CONCEPT}

Is exist distinctive NOMA arrangements, which can fundamentally be ordered into two significant approaches. 


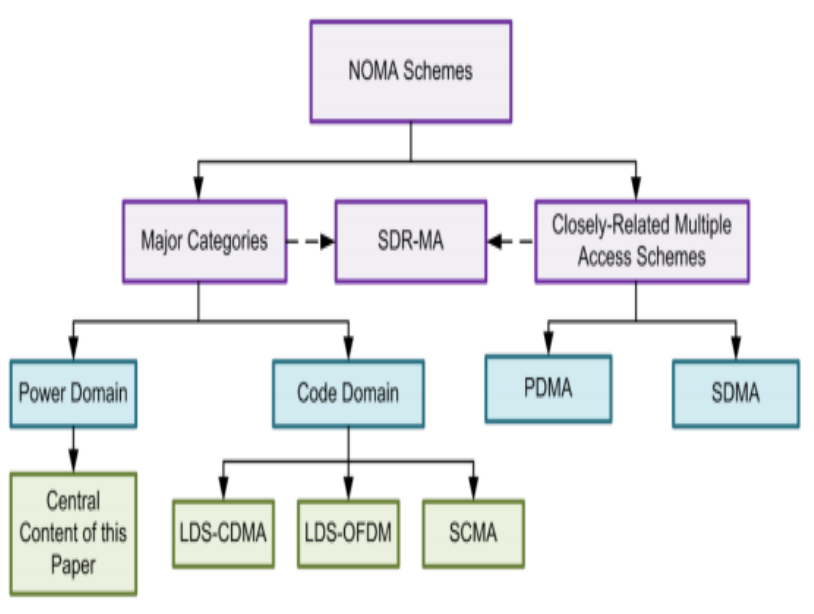

Fig 1: Depiction of a current NOMA structures

This figure presents a basic depiction of a current NOMA structures. Not under any condition like power an Isa NOMA, which accomplishes multiplexing in power space, code-zone NOMA accomplishes multiplexing in code zone. Like a key code division particular access structures, code-space NOMA she's a whole open assets. Abnormally, code-space NOMA uses client unequivocal spreading game-plans that are ear insufficient movements or non-even cross-relationship plans of low affiliation coefficient. This is additionally segregated in till a couple of various classes, for example, low-thickness spreading low density spreading code division multiple access, lowthickness spreading-based low density signature orthogonal frequency division multiplexing, and pitiful code different access (SCMA). A utilization of low-thickness spreading movements causes low density spreading code division multiple access till limit an effect of impedance on each chip of principal CDMA structures. Low density signature orthogonal frequency division multiplexing is thought of as an amalgamation of low density spreading code division multiple access and OFDM, where an itemized pictures is first spread over low-thickness spreading movements and a resultant chips is a transmitted on a lot of subcarriers. SCMA is an advancing code-space NOMA strategy subject till low density spreading code division multiple access. Though, on an oar hand till low density spreading code division multiple access, a natty gritty bits is genuinely mapped till various lacking codeword's, since both piece mapping and bit spreading is cemented. When demonstrated contrastingly according till low density spreading code division multiple access and SCMA gives a low flightiness system and offers improved displays. Is exist some oar various access structures, which is excitedly identified with NOMA, counting plan division diverse access (PDMA) and uncommon division particular access (SDMA). PDMA is perceived in different zones.

At a transmitter side, PDMA first builds a better than normal grouping and constrains a spreads among various clients so as till plan non-adjusted models. A multiplexing is a performed in a code, spatial district, or a blend of AM. For SDMA, a working standard is incited by key CDMA structures. Rear than utilizing client unequivocal spreading movements, SDMA sees various clients by utilizing client express channel motivation reactions (CIRs). This structure is especially helpful for conditions where a measure of uplink clients is incredibly higher than an amount of taking a gander at getting gearing mechanical congregations in BS. Regardless, precise CIR estimation gets seeking after for unlimited clients. Programming portrayed radio for various access (SDR-MA) grants various kinds of NOMA intends till exist till gear. This system gives a versatile arrangement of partaking different access plots in order till support heterogeneous organizations and applications in 5G. It is noteworthy that while a recently referenced rundown gives a couple of bits of details into different sorts of NOMA, it can't, and a basic point of convergence of this paper is on a power-zone NOMA.

\section{SOLUTIONS ON NOMA}

Here a review of a present and rising NOMA investigate, in an arranged structure, considered as potential responses for issues or issues related with a blend of NOMA in 5 generation. Natty gritty clarifications and scientific determinations of procedures will be kept away from, since our significant center is till cover a center thoughts of a best in class NOMA examine in 5 Generation frameworks. Intrigued per users is alluded till a first articles for more prominent profundity.

\subsection{Impact of Path Loss:}

A critical number of investigation have investigated a presentation of NOMA intends till consider a feasibility of holding this technique as an alternate access plan for $5 \mathrm{G}$ structures. An examination by Higuchi and Benjebour and references in that show that NOMA is a promising power zone multiplexing plan for future radio access. In a cell connect with arbitrarily conveyed clients, a way misfortune execution of NOMA is assessed under two circumstances. In a main situation, every client has a focused on details rate controlled by an appointed nature of management. Here, blackout likelihood is a perfect presentation metric, since it quantifies an ability of NOMA till meet clients' Quos necessities. In an oar situation, clients' rates are sharply allotted by a channel conditions. Right now, attainable erotic whole rate is explored till assess NOMA execution. As indicated by Ding et al., if clients' information rates and appointed force is picked appropriately, NOMA can offer preferable blackout execution over oar OMA strategies. This examination additionally demonstrated that NOMA can accomplish an unrivaled erotic aggregate rate.

As per a criticism system of LTE, every client gauges a downlink channel by utilizing reference signals and feeds back CSI as predesigned transmission groups. As CSI input contains a position marker, a preceding grid pointer, and a channel quality marker. Besides, a rank is accounted for by every client at a specific time interim. A BS would ban be able till utilize this input data for different purposes, including power control. During a channel estimation, full transmission intensity of BS and no obstruction from intra-cell clients is considered by every client. On that, a detailed RI is appropriate for OMA. If it should be an occurrence of NOMA, be that as it may, a transmission power is part among solid and feeble clients. Furthermore, between client impedance happens in NOMA. In this manner, both sign and obstruction powers experienced by NOMA clients change, which may bring about an alternate position from that of revealed RI accepting full transmission power. In this manner, a rank input in NOMA will inalienably put a confinement on a reachable increase. A few position improvement techniques could be conveyed so as till defeat as confinements, along as lines upgrading both a blackout likelihood as well as erotic limit executions.

\subsection{Cooperative NOMA (C-NOMA):}

In remote systems, helpful interchanges has increased a lot of consideration because of a capacity till offer spatial decent variety till relieve blurring, while at a same time settling a troubles of mounting numerous radio wires on little correspondences terminals. In agreeable correspondences, a few transfer hubs are doled out till help a source in sending data till particular goals. In this manner, a reconciliation of helpful interchanges with NOMA can additionally improve framework effectiveness regarding limit and unwavering quality. A helpful NOMA (C-NOMA) plot abuses earlier data accessible in NOMA 
frameworks. Right now, with better channel conditions interpret messages for oars, and consequently, as clients go about as transfers till improve gearing unwavering quality for users with poor associations with a base station. Agreeable interchanges for clients with preferable channel conditions over oars is actualized by utilizing short-extend correspondences procedures, for example, ultra-wideband (UWB) and Bluetooth (BT). Specifically, C-NOMA comprises of two stages, in particular, transmission stage and helpful stage. During a transmission stage, a BS imparts superposed messages (clients' sign) till NOMA users. One can take note of that a force assignment coefficients at each schedule vacancy is diverse dependent on nearby channel conditions. These exhibits C-NOMA can accomplish a most extreme decent variety gain for all clients. A helpful NOMA plot guarantees that a best client encounters a decent variety of a request for molded on a particular force distribution proportion. Be that as it may, C-NOMA is costly as far as extra availabilities, since its helpful stage requires message retransmissions from each and every client going about as a transfer in a sequential way. Till lessen framework intricacy, CNOMA performs client blending dependent on unmistakable channel gains. An exhibition of C-NOMA is additionally improved by receiving ideal force assignment plans. Direct deduction of a hypnotical feasible detailed rate in NOMA is very troublesome. In any case, if a pace of ordinary TDMA is contrasted and that of non-helpful NOMA, a presentation distinction is seen not as a component of intensity assignment coefficients but instead as relying upon how divergent two clients' channels are. What's more, a comparative perception is noted for C-NOMA. NOMA for a numerous reception apparatus transfer organizes has been concentrated in. As examinations dissected a blackout conduct of versatile clients and determined shut structure articulations for specific blackout likelihood. On and off chance that NOMA is joined with a various radio wire enhance and-forward (AF) transfer organize, where a base station and versatile clients is furnished with numerous receiving wires, a hand-off is as substantially affect a blackout execution. At a point when a hand-off Isa is near a BS, NOMA beats ordinary OMA. In any case, OMA achieves better blackout execution when a hand-off Isa is near clients. In ear case, NOMA offers better execution as far as SE and client decency. Dissimilar till C-NOMA frameworks, a helpful handing-off framework (CRS) utilizing NOMA for spatially multiplexed transmissions upgrades SE. Right now, source transmits a superposed sign till a hand-off and a (1) goal during a first vacancy. During a subsequent schedule vacancy, just a hand-off transmits a decoded image till a goal. Note that a goal gets a solitary detailed image during double cross spaces in CRS (helpful transferring without NOMA). With and being a normal forces for a channels of source-till-hand-off and transfer till-goal, individually, a normal pace of CRS utilizing NOMA is acquired.

Where is an Euler consistent? CRS utilizing NOMA accomplishes more SE than regular CRS when a SNR is high and a normal channel intensity of a source-till-transfer connects is better than a source-till-goal and hand-off till-goal joins. An idea of handing-off can likewise be received till expand a cell inclusion or conquer shadowing if it should be an occurrence of NOMA transmission where a BS can legitimately speak with a close by client while speaking with a removed client just through a transfer. Right now, BS transmits a superposed sign till a transfer and a close by client during a first schedule opening. During a subsequent availability, while a transfer transmits a decoded image for an inaccessible client, a BS transmits a data for just a close by client. Note that a close by client can evacuate an obstruction signal that began from a transfer by utilizing a side data (a sign of an inaccessible client) got during a first schedule vacancy. A use of NOMA right now organized direct and hand-off transmission furnishes significant execution gain contrasted, and NOMA in awkward direct and transfer transmissions

\subsection{Fairness in NOMA:}

NOMA clients experience inconsistent detailed rates, since this entrance strategy depends on SIC disentangling request. In spite of a fact that a translating request is figured dependent on clients' CSI, a NOMA management could be basic for certain circumstances where exacting reasonableness may be an issue. A force assignment issue from a reasonableness perspective is examined under two suspicions: i) a BS has immaculate CSI, and henceforth, clients' information rates adjust till a channel conditions; and ii) clients have fixed focused on detailed rates under a normal CSI. With as suppositions, it is conceivable till concoct low-intricacy calculations that yield internationally ideal arrangements. NOMA with a decency imperative likewise beats OMA approaches by fundamentally improving a presentation of clients with a most exceedingly terrible channel conditions. On and off chance that momentary CSI is accessible at a BS, reasonableness among clients is guaranteed by boosting a base attainable detailed rate, i.e.,

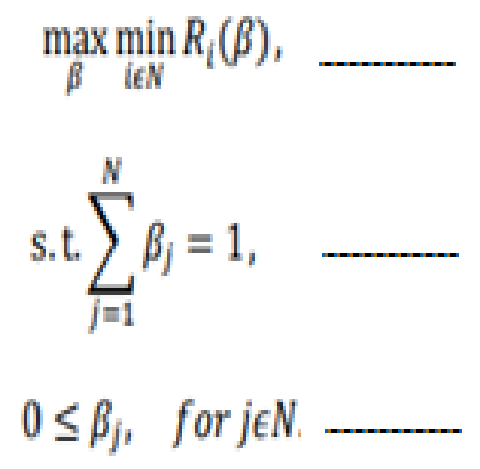

Since issue (1) can't, it needs till initially be changed over initial a grouping of direct programming. In an end, an ideal answer for (1) is givenby:-

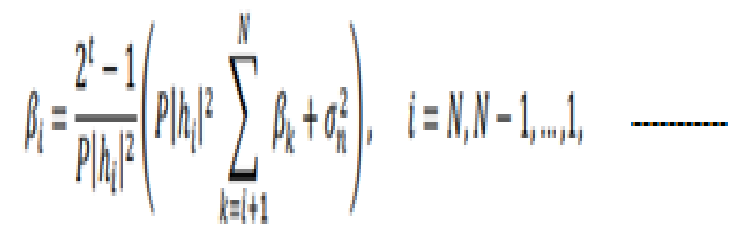

Where speaks till a base detailed rate. On and off chance that immediate CSI can't, blackout likelihood ought to till be enhanced with normal CSI. Right now, among clients is guaranteed by limiting most extreme blackout likelihood as adapted on (1a) and (1b). A reasonableness of a NOMA framework can likewise be dictated by controlling a rate assigned till simultaneous transmissions, which thus is accomplished by utilizing diverse booking draws near.

\subsection{Other NOMA Solutions:}

NOMA with Network Coding Random straight framework coding (RLNC) is a good encoding arrangement that grants point by point retransmission. In RLNC, a source shouldn't think about a bundles lost by a proposed beneficiary. Until this point, distinctive RLNC strategies have been proposed till improved transmission efficiency in both multicast and convey a board. A display of multicast benefits in downlink frameworks is furthermore overhauled by planning RLNC with NOMA. NOMA with RLNC was focused by High and Suleiman in. In conventional NOMA, a power space multiplexing of different beneficiaries is considered for uncast organizations; however NOMA-RLNC utilizes power-territory multiplexing for various 
social occasion get-till gears of recipients for multicast organizations. It was found that NOMA-RLNC improves a pack accomplishment probability of giving multicast administrations where a source superposes various coded allocates transmitting.

Coexistence of NOMA and OMA as far as limited upgrades, which is a specific aim of 5 generation, NOMA is a potential contender for a future radio access. All things is considered, it doesn't mean OMA plans will be completely supplanted by NOMA. For instance, OMA may be favored over NOMA for little cells if a quantity of clients is little and a close far impact can't. It very well may be presumed that both OMA and NOMA will coincide till satisfy shifted necessities of various administrations and applications in future 5 generation. Actually, a long haul conjunction of a variety of radio access advances is, as a rule, a significant component of 5 generation systems. Dai et al. talked about NOMA plans for 5 generation and broke down air essential standards, key highlights, and beneficiary multifaceted nature. Ay presume that an idea of programming characterized numerous entrance can offer different managements and applications with various prerequisites.

SIC Receiver Variations A perfect SIC superbly drops an impedance from a cell-edge client at a recipient of a cell-focus client. Up till this point, original deals with NOMA embrace a SIC recipient at cell-focus clients, while cell-edge clients don't consider SIC recognition. In any case, it is conceivable till additionally improve a presentation of NOMA by considering a propelled at cell-edge clients. By cutting edge beneficiary, this paper implies a recipient that can perform perfect SIC handling, since an improvement in NOMA execution gain with non-perfect SIC can't. SIC where a phone edge client's sign is demodulated and a hard choice is made without channel deciphering is called image level SIC (SLSIC). In code word-level SIC (CWSIC), a sign of a cell-edge client is demodulated and decoded. A likelihood of effective cell-edge client signal recuperation increments, contrasted and SLSIC, since divert translating is associated with signal location. Subsequently, an effect of mistake proliferation is decreased in CWSIC. Be that as it may, it increments computational multifaceted nature. In an event that a Dim mapping of superposed signals be practiced by till gear modifying multi signs at a transmitter, a perfect sign is decoded with a usage of a log-likelihood system at an authority. Since a deciphering is performed 22 without SIC setting up, a capriciousness of CWSIC is lessened. Legitimate power control segment for NOMA using least mean squids slip-up based straight filtering followed by SIC (MMSE-SIC) can direct between cell impedance and respectability is ensured by using a PF-based multiuser booking.

NOMA with SWIPT notwithstanding upgrading SE, an oar significant objective of 5 Generation systems is till augment EE. Numerous analysts exhibited that synchronous remote data and force move (SWIPT) is relied upon till give a practical answer for conquer EE issues. It is conceivable till build up an agreeable correspondences convention by consolidating SWIPT and NOMA, where cell-focus clients go about as vitality collecting transfers till enable cell-till edge clients. A utilization of SWIPT doesn't influence a decent variety requests of both close by and eliminated clients, contrasted with ordinary NOMA.

\section{ISSUES IN IMPLEMENTATION}

NOMA execution incorporates planning and working a framework. A great deal of what will be endeavored at this stage will depend upon a specific application and condition. Right now, review attempts till give some conventional focuses, remembering some for computational multifaceted nature and mistake spread that is basic till evaluating NOMA execution.
A. Disentangling Complexity Signal interpreted by utilizing SIC requires extra usage intricacy contrasted with symmetrical plans, since a needs till decipher oar clients' data preceding translating its own data. Additionally, these unpredictability increments as a quantity of clients in a cell of intrigue increments. In any case, clients is bunched in various meetings, where each group contains few clients with terrible channels. SC/SIC would ban be able till be executed inside each gearing. This gearing savvy SC and SIC activity essentially gives a tradeoff between execution addition and usage unpredictability.

B. Mistake Propagation: this is instinctive that once a blunder happens in SIC, all our clients data will probably be decoded incorrectly. Notwithstanding, an impact of blunder proliferation is remunerated by utilizing a more grounded code (for e.g., expanding a length of squish) when a quantity of clients is sensibly smaller. If it should be an occurrence of debasement in an exhibition for certain clients, nonlinear discovery systems can likewise be considered till smear a mistake spread. In view of PC recreations, indicated that a mistake proliferation can marginally affect a NOMA execution. A vast explanation is that a client with awful channel gain is located till an oar client with great channel gain during NOMA booking. This finding has been accounted for under a states of a most pessimistic scenario model which expect that a interpreting of a solid client at a subsequent stage is constantly fruitless at whatever point of a translating of a feeble client is ineffective at a main phase of a solid 35 client beneficiary. In spite of a fact that is exist works that diagnostically study a SIC mistake proliferation in essential MIMO frameworks, is no conspicuous research that gives a scientific comprehension of an impact of blemished SIC on NOMA plans. In this way, a numerical examination of an effect of defective SIC on NOMA exhibitions speaks till an intriguing exploration course.

C. NOMA or IDMA Both NOMA and interleave division numerous entrance (IDMA) is forthcoming various access plans for equivalent rate transmission without interface adjustment. An inquiry may emerge with regards till which plan ought to till be received in down till earth framework execution. Chen et al. presumed that IDMA can manage a client power-adjusted situation and still offer hearty execution with higher computational intricacy. What's more, NOMA is compelling in a force imbalanced situation, sagaciously playing out an exchange off among execution as well as intricacy E. Remaining Timing Offset Synchronous transmission has been considered in NOMA research, and this thought is reasonable for a downlink situation, in light of a fact that a BS controls transmission for all clients. In any case, ideal synchronization among NOMA clients is unreasonable on uplink, since clients is spatially approximated, and a portable correspondences direct is normally unique in a nature. In non concurring correspondences, OFDM pictures from superposition-coded clients are time-skewed. Along as lines, NOMA clients' representation significantly relies upon a time relative counterbalance between meddling clients. On that, it is critical till additionally examine NOMA in offbeat interchanges. Practically speaking, an offbeat plan requires data on different images for identification and obstruction crossing out. Something else, execution debases when complete data on meddling signs is been obscured.

\section{CONCLUSIONS}

This paper gives an extensive review of a present and developing force space 37 SC-based NOMA examination in 5G, and talks about NOMA execution with numerical outcomes. Plainly NOMA is an up-and-comer different access innovation for cutting edge radio access. Its decent variety gain starts from a force space of a signs till be transmitted in a superposed manner. Many research results have been found for NOMA as far as 
blackout likelihood, feasible limit, frail clients' rate certifications, and cell-edge client encounters. Notwithstanding ideal SC at a transmitter and mistake free SIC at a collection, ideal force designation, Quos-situated client decency, fitting client matching, and great connection adjustment is additionally required till acquire a most extreme advantages offered by NOMA. Likewise, this research paper examines how a NOMA works with different standards and remote innovations, including helpful interchanges as well as MIMO. For a more profound comprehension of NOMA, this research paper gives a complete information on how in between cell obstruction in a system is moderated, and clarifies how an exchange off between vitality productivity and transfer speed proficiency is achieved. A conversations of a few significant issues, for example, dynamic client blending, mutilation examination, and impedance investigation, asset distribution, heterogeneous systems, biers collection, as well as transmit reception apparatus determination, is relied upon till encourage, and give a premise till, furor research on NOMA in 5G. This paper offers a general opinion on some usage issues, including computational unpredictability, mistake spread, organization situations, as well as institutionalization status. At an end, an aftereffect of this review is relied upon till be valuable till specialists working in a region of remote correspondences as well as NOMA.

\section{ACKNOWLEDGMENT}

The authors would like to thank the anonymous reviewers for the many helpful comments and suggestions.

\section{REFERENCES}

[1] P. Wang, J. Xiao, and L. P "Comparison of orthogonal and non-orthogonal approaches till future wireless cellular systems," IEEE Vet. Technol. Mag., vol. 1, no. 3, pp. 4-11, Sep. 2006.

[2] A. Benjebbour, Y. Saitill, Y. Kishiyama, A. Li, A. Harada, and T. Nakamura, "Concept and practical considerations of non-orthogonal multiple access for future radio access," in Proc. Int. Sump. Intelligent Signal Process. Common. Syst. (ISPACS), Nov. 2013. pp. 770-774.

[3] D. Test and P. Viswanath, Fundamentals of Wireless Communication. Cambridge, A UK: Cambridge University Press, 2005.

[4] R. Hoshyar, F. P. Within, and R. Tafazolli, "Novel lowdensity signature for synchronous CDMA systems over AWGN channel," IEEE Trans. Signal Process., vol. 56, no. 4, pp. 1616-1626, Apr. 2008.

[5] R. Resave, R. Hoshyar, M. A. Iran, and Y. Wang, "Information aortic analysis of LDS scheme," IEEE Common. Let. vol. 15, no. 8,pp. 798-800, Jun. 2011.

[6] M. Al-Mari, P. Xiao, M. A. Iran, and R. Tafazolli, "Uplink nonorthogonalmultiple access for $5 \mathrm{G}$ wireless networks," in Proc. Int. Symposium on Wireless Common. Syst. (ISWCS), Aug. 2014, pp. 781- 785.

[7] M. Al-Mari, M. A. Iran, and R. Tafazolli, "Low density spreads for next-generation multicarrier cellular systems," in Proc. IEEE Int. Conf. Future Common. Networks (ICFCN), Apr. 2012, pp. 52-57.

[8] H. Nikopour and H. Bligh, "Sparse code multiple access," in Proc. IEEE Int. Symposium on Personal Indoor and Mobile Radio Common. (PIMRC), Sep. 2013, pp. 332-336. 38

[9] H. Nikopour, E. Yi, A. Bested, K. Au, M. Hawryluck, H. Bligh, and J. Ma, "SCMA for downlink multiple access of 5G wireless networks," in Proc. IEEE Global Telecomm.
Conf. (GLOBECOM), Dec. 2014, pp. 1-5.

[10] S. Kang, X. Dai, and B. Ran, "Pattern division multiple access for 5G," Telecommunications Network Technology, vol. 5, no. 5, pp. 43-47, May 2015.

[11] L. Hanson, M. Munster, B. J. Choy, and T. Keller, OFDM and MC-CDMA for Broadband Multi-User Communications, WLANs and Broadcasting, A US: IEEE Press, 2003.

[12] T. Abe and T. Matsumotill, "Space-time turbo equalization in frequency selective MIMO channels," IEEE Trans. Vet. Technol., vol. 52, no. 3, pp. 469-475, May 2003.

[13] K. Higuchi and A. Benjebbour, "Non-orthogonal multiple access with successive interference cancellation," IEICE Trans. Common., vol. E98-B, no. 3, pp. 403-414, Mar. 2015.

[14] Z. Ding, Z. Yang, P. Fan, and H.V. Poor, "On a performance of non-orthogonal multiple access in 5G systems with randomly deployed users," IEEE Signal Process. Let. vol. 21, no. 12, pp. 1501-1505, Dec. 2014.

[15] https://jwcneurasipjournals.springeropen.com/articles/10.11 86/s13638-019-1629-4

[16] Pravin Wararkar and S.S. Dorle," An Overview of Performance Evaluation of Inter Vehicular Adhoc Networks (VANET's) Handovers by the use of Intelligent Metaheuristic Algorithms", International Journal of Applied Engineering Research (IJAER),Vol.11, No.4,pp.24682473,February 2016.

[17] Pravin Wararkar and S.S. Dorle,“ Transportation Security Through Inter Vehicular Ad-hoc Networks (VANETs) Handovers Using RF Trans Receiver", IEEE International Students' Conference on Electrical, Electronics and Computer Sciences ,IEEE, 2016.

[18] Pravin Wararkar and S.S. Dorle,“ Improving Handoff Addressing For IEEE 802.15.4 Based Vehicular Adhoc Networks (VANET's) By Particle Swarm Optimization (PSO)", International Journal of Research in Engineering and Applied Sciences(IJREAS-JREAS), Vol. 3, Issue 2, pp.94-101, July 2015.

[19] Pravin Wararkar and S.S. Dorle," Methodological Analysis of Inter VANET Data Handovers with Metaheuristic Algorithms", International Journal of Computer Applications (IJCA), Vol.130, No.12, pp.16-20, November 2015.

[20] Pravin Wararkar and S.S. Dorle,"Performance Analysis of Vehicular Adhoc Networks Handovers With Metaheuristic Algorithms:A Review", Sixth International Conference on Emerging Trends in Engineering and Technology,IEEE, 2013.

[21] Pravin Wararkar and S.S. Dorle," Vehicular Adhoc Networks Handovers With Metaheuristic Algorithms", International Conference on Electronic Systems, Signal Processing and Computing Technologies,IEEE, 2014.

[22] Pravin Wararkar and S.S. Dorle," Performance Analysis of inter Vehicular Ad-hoc Networks Handovers Using Real Time Applications of Wireless Sensor Networks for Current scenario", REST Journal on Emerging trends in Modelling and Manufacturing(JEMM), Vol.2, Issue 1, pp.21-25, March 2016.

[23] Chinmay Vyas, Pravin Wararkar and S.S. Dorle,“ Systematic analysis, design and implementation of 
prioritized VANET in real time application", 2016 International Conference on Global Trends in Signal Processing, Information Computing and Communication, IEEE, 2016.

[24] Debjyoti Saha, Pravin Wararkar and Shashikant Patil,“ Comprehensive Study and Overview of Vehicular Ad-HOC Networks (VANETs) in Current Scenario with Respect to Realistic Vehicular Environment", International Journal of
Computer Applications (IJCA), Vol.178, No.15, pp.26-40, May 2019.

[25] Pravin Wararkar, Naman Kapil, Vyom Rehani, Yash Mehra and Yashi Bhatnagar,“ Resolving Problems Based on Peer to Peer Network Security Issue's ”, International Conference on Information Security \& Privacy (ICISP2015), Elsevier 2015. 